\title{
Study on Health and Life Habits, Oxidative Stress, and Antioxidant Capacity Among Female Care Workers and Female General Workers
}

\author{
Yukiko Kawano $^{1}$, Shinichi Demura ${ }^{2}$, Yoshiharu Tanaka ${ }^{3} \&$ Yoshimasa Matsuura $^{3}$ \\ ${ }^{1}$ Department of Child Education, Kobe College of Education, Japan \\ ${ }^{2}$ College of Human and Social Sciences, Kanazawa University, Japan \\ ${ }^{3}$ Faculty of Liberal Arts and Sciences, Osaka Prefecture University, Japan \\ Correspondence: Yukiko Kawano, Department of Child Education, Kobe College of Education, Japan. Tel: \\ 81-786-113-351.
}

Received: June 2, 2020

Accepted: July 20, 2020

Online Published: August 7, 2020

doi:10.20849/ijsn.v5i3.785

URL: https://doi.org/10.20849/ijsn.v5i3.785

\begin{abstract}
Background: Care workers have been considered to have higher work-related stress than general workers, which can have a considerable effect on their life habits. Hence, psychological and physiological stress levels should be examined. While several studies have investigated psychological stress among care workers, none have examined physiological stress. In addition, very few studies have been investigated for the relationship between life habits and physiological stress.
\end{abstract}

Aims: This study aimed to examine the relationship between health and life habits and physiological stress among female care workers and general workers and identify differences therein.

Methods: Subjects included 30 care workers (average age: 49.8) and 33 general workers (average age: 51.9). No significant difference in their age was noted. The "Diagnostic Inventory of Health and Life Habit" developed by Tokunaga was used to examine health and life habits. Physiological stress was examined by measuring diacron-reactive oxygen metabolites (d-ROMs) and biological antioxidant potential (BAP) using FRAS4 manufactured by WISMERLL Co., Ltd. during the subjects' working hours.

Results: Care workers were taller, heavier, woke up later, and slept longer than general workers. Both care workers and general workers had average health and life habit parameter scores that fell within the "moderate" and "slightly low" categories, with no significant differences between both groups. Both care workers and general workers were judged as "caution required" which indicated undesirable health and life habits. No significant differences in d-ROMs and BAP were observed between both groups, many of whom fell under the "high oxidative stress" category for d-ROMs and "borderline" for BAP. Regarding the relationship between d-ROMs and BAP values and health and life habit parameters, our results found some relationship between exercise behaviors and d-ROMs only among care workers.

Conclusion: General workers and care workers included herein had similarly poor health levels and undesirable life habits. Moreover, both groups had high d-ROMs and normal BAP levels in average, with no significant differences in both indexes. Several care workers with high d-ROMs tended to not participate in sport activities or exercise and did not expect to profit from them.

Keywords: female care workers, female general workers, psychological stress, physiological stress, health and life habits, oxidative stress, antioxidant capacity

\section{Introduction}

Today, Japan continues to face declining birth rates and increasing aging population, which has led to a yearly growth in the number of senior citizens needing care. Moreover, in 2007, the Care Work Foundation showed that the mean turnover rate of care workers $(21.6 \%)$ was higher than the overall mean turnover rate within the industry (15.4\%).

Nara et al. reported that care workers working at special elderly nursing homes had a short job tenure (over half of the workers had below 5 years of experience) and high turnover rates. Accordingly, disturbed life habits often due to irregular scheduling and night shifts (Nara et al., Okamura et al.), as well as considerable physical and mental burden related to lifting assistance or bathing assistance (Kawano et al.), have been thought to promote high turnover rates among care workers. 
Nakao revealed that nurses who worked like care workers suffered from similar mental and physical fatigue and that shift work considerably affected the human body and life habits.

Okazaki et al. surveyed life habits of nurses and care workers and clarified actual circumstances, such as weight gain, poor dietary balance, increased snacking, unsteady life habits, and skipping breakfast.

Ono et al. reported that night shifts promoted not only irregular sleep-wake patterns but also irregular meal schedules and bowel movement timing among care workers.

The aforementioned studies suggest that irregular working hours and work overload of care workers disturb their life habits and promote poor physical condition owing to irregular mealtimes, lack of exercise and sleep, weight gain by snacking, and poor nutritional balance (Okazaki et al.). Thus, care workers experience severe physical and mental stress and may have difficulty with continuing labor, which subsequently leads to resignation.

A considerable number of studies on occupational stress and life habits (Ono et al., Takenaka.) have shown that stress and life habits are closely related. Several survey studies regarding occupational stress for reducing turnover rates among nurses and care workers (Kawano et al., Inaba., et al., Kwiatkowski., Maslach., Hayashi et al.) have also been conducted. According to the labor security hygiene findings of Japanese Ministry of Health, Labor and Welfare (2017), approximately $60 \%$ of workers experience considerable stress during work.

Stress evaluation among general workers has been widely performed through interviews, question surveys and psychiatric tests (Ministry of Health, 2005., Shimomitu., Onoda et al.)

However, such methods have problems with low reliability given that they are based on subjective input of the individual (Fukuda.). Therefore, to objectively evaluate stress, physiological stress indexes, such as diacron-reactive oxygen metabolites (d-ROMs) (Wayner et al., Seki.) and biological antioxidant potential (BAP) (Alberti et al., Dohi et al.) in the blood, have currently been used.

Nojima et al. examined fatigue among disease-free subjects, those with chronic fatigue syndrome, those with industrial fatigue, those with high mental workload, those who survived the Great East Japan Earthquake, and other general subjects using d-ROMs and BAP. Moreover, they suggested that d-ROMs accumulation is associated with various life habit-related diseases and promotes aging. Hence, the evaluation of d-ROMs (Wayner et al., Seki.) and BAP (Alberti et al., Dohi et al.) values can be a useful method for objectively evaluating mental and physical fatigue.

Kuragaki clarified that the d-ROMs and BAP are effective as preventive medicine of risk factors on health along with life habits and work environment, and they have a possibility to contribute to people's health.

According to Komatsu et al. and Mimura et al., d-ROMs are especially influenced by diet, physical activity, and life habits and d-ROMs and BAP are effective tools for objectively evaluating stress.

However, studies focusing on stress among care workers based on physiological markers such as d-ROMs and BAP levels are scarce. Casado et al., measured the activities of superoxide dismutase, catalase and the concentration of malondialdehyde as stress markers for care workers but did not use d-ROMs or BAP levels.

Given that clarifying the relationship between work stress and life habits among care workers may contribute toward reducing turnover rates, the present study aimed to examine differences in health, life habits, and physiological stress (d-ROMs and BAP), as well as their relationship, among female care workers and general workers.

\section{Method}

\subsection{Subjects}

Participants included 30 female care workers with Home Helper certification examinations over Level 2 (hereafter, care workers) and 33 female general workers (hereafter, general workers). General workers' occupations are categorized as clerical workers, medical workers, university staff, restaurant staff, and full-time homemakers. Written informed consent was obtained from all subjects after explaining in detail the purpose of this study.

\subsection{Survey on Physique and Sleeping}

Lifestyle items (average in a week), including awakening time, bedtime, and sleeping hours, were surveyed in addition to height and weight. Body mass index (BMI) was then calculated.

\subsection{Survey on Health and Lifestyle}

The National Health and Nutrition Survey (2018) developed by the Ministry of Health, Labor and Welfare has been widely used in Japan. However, its contents are limited to dietary habits, sleep conditions, favorite food, dental condition, and life and social habits. In contrast, the Diagnostic Inventory of Health and Life Habit 
(hereafter, DIHAL.2) developed by Tokunaga has been generally used to evaluate health and life habits among middle school-age to elderly individuals, as well as among various occupations, given that it can comprehensively and objectively evaluate health and life habit factors. The validity of the question items has also been well examined. Therefore, the current study used the DIHAL.2 to evaluate the health and life habits of our subjects.

The DIHAL. 2 consists of 47 question items under four parameters, health status, movement habits, meal habits, and rest habits (hereafter health and life habit parameters), which are subdivided into 12 sub-factors ([1] - [12]). Subjects answered each question using a 5-point Likert-type scale (1, very low; 2, slightly low; 3, moderate; 4 , slightly good; 5, very good). The following outlines the sub-factors and number of questions therein in parentheses.

1. Health status (12): [1] Physical health status (4), [2] Mental health status (4), and [3] Social health status (4)

2. Movement habits (8): [4] Exercise behavior and conditions (5) and [5] Exercise awareness (3)

3. Meal habits (13): [6] Dietary balance (7), [7] Meal regularity (4), and [8] Favorite food items (2)

4. Rest habits (14): [9] Rest (3), [10] Sleep regularity (3), [11] Sleep sufficiency (4), and [12] Stress coping (4)

Tokunaga considered the health status, movement, meal, and rest parameters to represent the health and life habit parameters. Accordingly, a higher total score in all four parameters suggests that the subject has a healthy and desirable life habits. Table 1 shows the total scores for all four parameters.

Table 1. Total scores for the health and life habit parameters and judgement

\begin{tabular}{llllll}
\hline \multirow{2}{*}{\multicolumn{1}{c}{ Parameters }} & \multicolumn{5}{c}{ Judgment } \\
\cline { 2 - 6 } & $\begin{array}{l}1 . \\
\text { (Very low) }\end{array}$ & $\begin{array}{l}\text { (Slightly low) } \\
\text { (. Health (12 questions) }\end{array}$ & $\begin{array}{l}3 . \\
\text { (Moderate) }\end{array}$ & $\begin{array}{l}\text { (Slightly good) } \\
\text { (Very good) }\end{array}$ \\
\hline 2. Movement (8 questions) & $8-18$ & $33-38$ & $39-44$ & $45-50$ & $51-60$ \\
\hline 3. Meal (13 questions) & $13-32$ & $33-41$ & $25-31$ & $32-37$ & $38-40$ \\
\hline 4. Rest (14 questions) & $14-29$ & $30-37$ & $32-50$ & $51-58$ & $59-65$ \\
\hline
\end{tabular}

\subsection{Indexes of Physiological Stress (Oxidative Stress and Antioxidant Capacity) and Their Measurement}

Recently, oxidative stress (d-ROMs) and antioxidant capacity (BAP) have been used to objectively evaluate stress (Nagata et al., Hatakeyama et al.). d-ROMs have been shown to be affected by lifestyle factors, such as diet and physical activity (Komatu et al., Mimura et al.)

Therefore, oxidative stress among disease-free individuals may reflect initial signs (pre-symptomatic state) of life habit-related diseases that could progress without subjective symptoms. Particularly, simultaneous measurement of d-ROMs and BAP can allow for an accurate comprehensive assessment of oxidative stress (Seki.).

Nagata et al. reported that d-ROMs reflect internal factors (heredity, disease, and sleep disorders) of life habit-related diseases, while BAP reflects external factors, such as dietary content (amount and quality). Hence, we determined that d-ROMs and BAP measurement was the most appropriate approach for evaluating physiological stress.

d-ROMs and BAP can be measured in about 10 min using small amounts of blood $(20 \mu \mathrm{L})$ obtained from the fingertips on a dedicated analytical equipment [Free Radical Analytical System 4 (FRAS4), manufactured by WISMERLL Co., Ltd.].

FRAS4 is equipped with a centrifuge, a spectrometer, and a printer. After piercing a fingertip using a lancet and obtaining blood into the designated container, bleeding is stopped using a hemostatic seal. Blood cell constituents and plasma are then separated using the centrifuge, after which $10 \mu \mathrm{L}$ of plasma is mixed into the container with a reagent exclusive for BAP or d-ROMs using a designated pipette. The container is then placed into the spectrometer for BAP or d-ROMs measurement. The machine then prints out measured values of BAP and d-ROMs. d-ROMs and BAP were evaluated based on the criteria presented in Tables 2 and 3. 
Table 2. Oxidative stress (d-ROMs test) and antioxidant capacity (BAP test) evaluation

\begin{tabular}{llll}
\hline $\begin{array}{l}\text { The oxidative stress measurement (d-ROMs test) } \\
\text { Unit: U.CARR }\end{array}$ & \multicolumn{2}{c}{$\begin{array}{l}\text { The antioxidant capacity measurement (BAP test) } \\
\text { Unit: } \mu \text { mol/ }\end{array}$} \\
\hline Normal & $200-300$ & Optimum value & Over 2200 \\
Borderline & $301-320$ & Borderline & $2000-2199$ \\
\hline Mild oxidative stress & $321-340$ & Antioxidant capacity is short a little & $1800-1999$ \\
Moderate oxidative stress & $341-400$ & Antioxidant capacity is short & $1600-1799$ \\
\hline High oxidative stress & $401-500$ & Antioxidant capacity is considerably & $1400-1599$ \\
Very high oxidative stress & Over 501 & short & Under 1399
\end{tabular}

${ }^{*} \mathrm{U}$. CARR is an arbitrary unit named after its developer: $1 \mathrm{U}$. CARR $=0.08 \mathrm{mg} / 100 \mathrm{~mL} \mathrm{H}_{2} \mathrm{O}_{2}$ equivalent

Table 3. Balance between oxidative stress and antioxidant capacity (evaluation matrix)

\begin{tabular}{llll}
\hline Oxidative stress (normal) & Antioxidant capacity (normal) = Optimum state & \\
\hline Oxidative stress (high) & Antioxidant capacity (normal) = Oxidative stress is & $\begin{array}{l}\text { Potential for problems in } \\
\text { excessive over antioxidant capacity }\end{array}$ & the future \\
\hline Oxidative stress (normal) & $\begin{array}{l}\text { Aantioxidant capacity (low) }=\text { Overall immune system } \\
\text { is weakened }\end{array}$ & $\begin{array}{l}\text { Potential for chronic } \\
\text { diseases }\end{array}$ \\
\hline Oxidative stress (high) & Aantioxidant capacity (low) = Immune function might & Special caution required \\
& & be weakening & \\
\hline
\end{tabular}

\subsection{Survey and Measurement Periods}

d-ROMs and BAP were measured during working hours in May 2016. Age, height, weight, awakening time, bedtime, and sleep duration were filled out using a self-administered questionnaire before d-ROMs and BAP measurement, while DIHAL.2 scores were determined using a self-administered questionnaire after d-ROMs and BAP measurement.

This study was approved by Kanazawa University Research Ethics Committee (Approval number 2012-19).

\subsection{Statistical Analysis}

Differences in the average value of each variable were determined using unpaired t-tests, while differences in the frequency of normal, borderline, and other ratings for d-ROMs and BAP test results were determined using the $\chi^{2}$ test. Relationships between variables were examined using Pearson's correlation coefficient and Kendall's rank correlation coefficient. The significance level during statistical hypothesis testing was set at 5\% and was controlled using the Bonferroni method.

\section{Results}

Table 4 summarizes the age, physical characteristics (height, weight, and BMI), awakening time, bedtime, sleep duration, d-ROMs, and BAP of the care workers and general workers. Care workers had a mean age of 49.8 years, height of $159.0 \mathrm{~cm}$, weight of $56.4 \mathrm{~kg}$, BMI of 22.3, awakening time of 7:10 am, bedtime of 11:30 pm, and sleep duration of $7 \mathrm{~h}$ and $40 \mathrm{~min}$. General workers had a mean age of 51.9 years, height of $155.2 \mathrm{~cm}$, weight of $52.2 \mathrm{~kg}$, BMI of 21.7, awakening time of 6:30 am, bedtime of 11:22 pm, and sleep duration of $7 \mathrm{~h}$ and $08 \mathrm{~min}$.

No significant difference in age was observed. Care workers were significantly taller and heavier, woke up later, and slept longer than general workers.

Care workers and general workers had a mean d-ROMs value of 452.4 and 414.4 U. CARR, respectively, with both groups falling into "high oxidative stress" category (Table 2). Care workers and general workers had a mean BAP value of 2060.3 and $2032.6 \mu \mathrm{mol} / \mathrm{L}$, respectively, with both groups falling into the "borderline" category (Table 2).

No significant differences in d-ROMs and BAP were observed between care workers and general workers. 
Table 4. Age, physical characteristics (height, weight, and BMI), awakening time, bedtime, sleeping duration, d-ROMs, and BAP of the care workers and general workers

\begin{tabular}{|c|c|c|c|c|c|c|c|c|c|c|c|c|c|}
\hline & \multicolumn{5}{|c|}{ Care workers $(n=30)$} & \multicolumn{5}{|c|}{ General workers $(n=33)$} & \multirow[t]{2}{*}{$\mathrm{t}$} & \multirow[t]{2}{*}{$\mathrm{p}$} & \multirow[t]{2}{*}{$\mathrm{d}$} \\
\hline & M & $\mathrm{SD}$ & $\mathrm{CV}$ & MAX & $\mathrm{MIN}$ & M & $\mathrm{SD}$ & $\mathrm{CV}$ & MAX & $\mathrm{MIN}$ & & & \\
\hline Age & 49.8 & 10.0 & 20.1 & 72.0 & 29.0 & 51.9 & 11.5 & 22.2 & 68.0 & 21.0 & 0.736 & 0.465 & 0.19 \\
\hline $\begin{array}{l}\text { Height } \\
\text { (cm) }\end{array}$ & 159.0 & 5.9 & 3.7 & 171.0 & 150.0 & 155.2 & 5.3 & 3.4 & 167.0 & 145.0 & 2.586 & $0.012 *$ & 0.66 \\
\hline $\begin{array}{l}\text { Weight } \\
(\mathrm{kg})\end{array}$ & 56.4 & 8.3 & 14.7 & 75.0 & 42.0 & 52.2 & 6.2 & 11.8 & 62.0 & 38.5 & 2.281 & $0.026^{*}$ & 0.58 \\
\hline BMI & 22.3 & 3.2 & 14.2 & 30.2 & 16.4 & 21.7 & 2.8 & 12.7 & 27.6 & 16.4 & 0.851 & 0.398 & 0.22 \\
\hline $\begin{array}{l}\text { Awakening } \\
\text { time }\end{array}$ & $\begin{array}{l}7: 10 \\
\text { am }\end{array}$ & $55 \mathrm{~min}$ & 12.8 & $\begin{array}{l}\text { 9:00 } \\
\text { am }\end{array}$ & $\begin{array}{l}5: 30 \\
\text { am }\end{array}$ & $\begin{array}{l}6: 30 \\
\text { am }\end{array}$ & $58 \mathrm{~min}$ & 14.8 & $\begin{array}{l}9: 30 \\
\text { am }\end{array}$ & $\begin{array}{l}4: 40 \\
\mathrm{am}\end{array}$ & 2.886 & $0.005^{*}$ & 0.74 \\
\hline $\begin{array}{l}\text { Sleeping } \\
\text { duration }\end{array}$ & $\begin{array}{l}7 \mathrm{~h} \\
40 \mathrm{~min}\end{array}$ & $65 \mathrm{~min}$ & 14.2 & $\begin{array}{l}10 \mathrm{~h} \\
00 \mathrm{~min}\end{array}$ & $\begin{array}{l}5 \mathrm{~h} \\
30 \mathrm{~min}\end{array}$ & $\begin{array}{l}7 \mathrm{~h} \\
08 \mathrm{~min}\end{array}$ & $59 \mathrm{~min}$ & 13.9 & $\begin{array}{l}9 \mathrm{~h} \\
30 \mathrm{~min}\end{array}$ & $\begin{array}{l}5 \mathrm{~h} \\
00 \mathrm{~min}\end{array}$ & 2.055 & $0.044^{*}$ & 0.53 \\
\hline $\begin{array}{l}\text { d-ROMs } \\
\text { (U.CARR) }\end{array}$ & 452.4 & 108.0 & 23.9 & 715.0 & 244.0 & 414.4 & 124.6 & 30.1 & 674.0 & 204.0 & 1.829 & 0.072 & 0.47 \\
\hline $\begin{array}{l}\text { BAP } \\
(\mu \mathrm{mol} / 1)\end{array}$ & 2060.3 & 184.8 & 9.0 & 2537.0 & 1845.0 & 2032.6 & 153.9 & 7.6 & 2292.0 & 1697.0 & 0.845 & 0.402 & 0.22 \\
\hline
\end{tabular}

M: Mean value, SD: standard deviation, CV: Coefficient of Variation, MAX: maximum value, MIN: minimum value, $t$ : statistic-t, $p$ : appearance probability, $d$ : effect size (Cohen's d)

*: $\mathrm{p}<0.05$

d- ROM: diacron-Reactive Oxygen Metabolites, BAP: Biological Antioxidant Potential

Table 5 summarizes the scores for the four health and lifestyle habit parameters among care workers and general workers.

All four health and life habit parameters among care workers and general workers fell within "moderate" and "slightly low" categories, which indicates criteria 3 and 2, respectively (see Table 1). No significant differences in all four parameters were observed between care workers and general workers.

Table 5. Scores for the four health and life habit parameters among care workers and general workers

\begin{tabular}{llllllllllllll}
\hline Parameters & \multicolumn{4}{c}{ Care workers $(\mathrm{n}=30)$} & \multicolumn{5}{c}{ General workers $(\mathrm{n}=33)$} & $\mathrm{t}$ & $\mathrm{p}$ & $\mathrm{d}$ \\
\cline { 2 - 11 } & $\mathrm{M}$ & SD & CV & MAX & MIN & M & SD & CV & MAX & MIN & & & \\
\hline 1.Health & 39.7 & 7.1 & 17.8 & 55.0 & 23.0 & 40.4 & 5.9 & 14.6 & 57.0 & 29.0 & 0.400 & 0.691 & 0.10 \\
\hline 2.Movement & 23.9 & 6.8 & 28.6 & 38.0 & 13.0 & 25.0 & 5.1 & 20.5 & 36.0 & 12.0 & 0.696 & 0.489 & 0.18 \\
\hline 3.Meal & 42.6 & 8.3 & 19.4 & 61.0 & 26.0 & 41.7 & 6.6 & 15.8 & 57.0 & 29.0 & 0.491 & 0.625 & 0.13 \\
\hline 4.Rest & 42.8 & 10.6 & 24.9 & 63.0 & 24.0 & 45.6 & 7.3 & 15.9 & 64.0 & 30.0 & 1.163 & 0.250 & 0.30 \\
\hline
\end{tabular}

M: Mean value SD: standard deviation CV: Coefficient of Variation MAX: maximum value MIN: minimum value t: statistic-t p: appearance probability d: effect size (Cohen's d)

*: $\mathrm{p}<\alpha / 4=0.0125$ 
Table 6 shows the correlation coefficients and partial correlation coefficients for the relationship between d-ROMs and BAP and physical characteristics, awakening time, bedtime, and sleeping duration among care workers and general workers

Among care workers, d-ROMs showed significant correlation with weight and BMI.

Among general workers, however, d-ROMs was significantly correlated with weight, awakening time, and bedtime, whereas BAP was significantly correlated with height and awakening time and negatively correlated with BMI.

Table 6. Correlation coefficients and partial correlation coefficients of d-ROMs, BAP, physique, awakening time, bedtime, and sleeping duration in care workers and general workers

\begin{tabular}{|c|c|c|c|c|c|c|c|c|}
\hline & \multicolumn{4}{|c|}{ Correlation coefficient } & \multicolumn{4}{|c|}{ Partial correlation coefficient } \\
\hline & \multicolumn{2}{|c|}{ d-ROMs } & \multicolumn{2}{|c|}{ BAP } & \multicolumn{2}{|c|}{ d-ROMs } & \multicolumn{2}{|c|}{ BAP } \\
\hline & $\begin{array}{l}\text { Care } \\
\text { Workers }\end{array}$ & $\begin{array}{l}\text { General } \\
\text { Workers }\end{array}$ & $\begin{array}{l}\text { Care } \\
\text { Workers }\end{array}$ & $\begin{array}{l}\text { General } \\
\text { Workers }\end{array}$ & $\begin{array}{l}\text { Care } \\
\text { Workers }\end{array}$ & $\begin{array}{l}\text { General } \\
\text { Workers }\end{array}$ & $\begin{array}{l}\text { Care } \\
\text { Workers }\end{array}$ & $\begin{array}{l}\text { General } \\
\text { Workers }\end{array}$ \\
\hline Age & 0.057 & 0.073 & -0.057 & -0.155 & & & & \\
\hline Height & 0.097 & 0.306 & -0.205 & $0.409^{*}$ & 0.134 & 0.324 & -0.254 & $0.393^{*}$ \\
\hline Weight & $0.377^{*}$ & $0.380^{*}$ & 0.012 & -0.151 & $0.439 *$ & $0.377^{*}$ & -0.013 & -0.144 \\
\hline BMI & $0.363^{*}$ & 0.204 & 0.130 & $-0.375^{*}$ & $0.382^{*}$ & 0.196 & 0.121 & $-0.359 *$ \\
\hline Awakening time & -0.136 & $0.365 *$ & 0.068 & $0.451 *$ & -0.124 & $0.387^{*}$ & 0.052 & $0.434 *$ \\
\hline Bedtime & -0.096 & $0.537^{*}$ & -0.109 & 0.089 & -0.083 & $0.542 *$ & -0.239 & 0.083 \\
\hline Sleeping duration & -0.006 & -0.279 & 0.179 & 0.329 & -0.048 & -0.272 & 0.262 & 0.315 \\
\hline
\end{tabular}

$*: \mathrm{p}<0.05$

Table 7 summarizes the frequency of normal (optimum value), borderline, and other ratings, for d-ROMs and BAP test results for care workers and general workers based on Tables 2, 3 and 4.

Most of the care workers and general workers were determined to be stressed $(90 \%$ and $73 \%$, respectively), with only a part of them having normal or borderline d-ROMs levels.

More than half of care workers and general workers (57\% and 61\%, respectively) had either optimum or borderline BAP values.

Table 7. Frequency (\%) of normal (optimum value), borderline, and other ratings by d-ROMs and BAP tests in care workers and general workers

\begin{tabular}{clllll}
\hline & & Care workers $(\mathrm{n}=30)$ & General workers $(\mathrm{n}=33)$ & $\mathrm{X}^{2}$ & $\mathrm{p}$ \\
\hline d-ROMs (\%) & Normal & $1(3.3)$ & $7(21.2)$ & 4.544 & 0.103 \\
& Borderline & $2(6.7)$ & $2(6.1)$ & & \\
& Other ratings & $27(90.0)$ & $24(72.7)$ & & \\
\hline BAP (\%) & Optimum value & $7(23.3)$ & $4(12.1)$ & 2.065 & 0.356 \\
& Borderline & $10(33.3)$ & $16(48.5)$ & & \\
& Other ratings & $13(43.3)$ & $13(39.4)$ & & \\
\hline
\end{tabular}

$\chi^{2}$ : Chi-squared test, $\mathrm{p}$ : appearance probability

$*: \mathrm{p}<0.05$

To examine the relationship between d-ROMs and health and life habit parameters, we categorized the d-ROMs into three ranking parameters (normal, borderline, and others ratings) and each health and life habit parameters into three ranking parameters excellent, moderate, and low that corresponded to the parameters very 
good/slightly good, moderate, and slightly low/very low, respectively (Table 1). Table 8 shows Kendall's rank correlation coefficients for care workers and general workers separately. Among care workers, our results showed a significant correlation only between movement habits and d-ROMs. No significant relationship was found between BAP and any health and life habit parameters among both care workers and general workers (data not shown).

Table 8. Rank correlation coefficients showing the relationship between d-ROMs and health and life habit parameters in care workers and general workers

\begin{tabular}{|c|c|c|c|c|c|c|c|c|c|c|c|c|c|c|c|}
\hline \multirow{3}{*}{ Parameters } & & \multicolumn{4}{|c|}{ Care Workers $(\mathrm{n}=30)$} & \multicolumn{7}{|c|}{ General Workers $(\mathrm{n}=33)$} & \multirow[b]{3}{*}{$\chi^{2}$} & \multirow{3}{*}{\multicolumn{2}{|c|}{$\mathrm{r}_{\mathrm{k}}$}} \\
\hline & & \multicolumn{4}{|c|}{ d-ROMs } & \multicolumn{7}{|c|}{ d-ROMs } & & & \\
\hline & & normal & borderline & other ratings & total & $\chi^{2}$ & $\mathrm{p}$ & $\mathrm{r}_{\mathrm{k}}$ & normal & borderline & other ratings & total & & & \\
\hline \multirow{4}{*}{ Health } & Excellent & 0 & 0 & 6 & 6 & & & & 1 & 1 & 6 & 8 & & & \\
\hline & Moderate & 0 & 1 & 11 & 12 & 2.130 & 0.712 & -0.201 & 3 & 1 & 8 & 12 & 1.924 & 0.750 & 0.063 \\
\hline & Low & 1 & 1 & 10 & 12 & & & & 3 & 0 & 10 & 13 & & & \\
\hline & Total & 1 & 2 & 27 & 30 & & & & 7 & 2 & 24 & 33 & & & \\
\hline \multirow{4}{*}{ Movement } & Excellent & 0 & 0 & 5 & 5 & & & & 0 & 0 & 4 & 4 & & & \\
\hline & Moderate & 1 & 2 & 4 & 7 & 10.952 & $0.027^{*}$ & 0.270 & 4 & 2 & 7 & 13 & 5.737 & 0.220 & 0.055 \\
\hline & Low & 0 & 0 & 18 & 18 & & & & 3 & 0 & 13 & 16 & & & \\
\hline & Total & 1 & 2 & 27 & 30 & & & & 7 & 2 & 24 & 33 & & & \\
\hline \multirow{4}{*}{ Meals } & Excellent & 0 & 0 & 6 & 6 & & & & 1 & 0 & 2 & 3 & & & \\
\hline & Moderate & 1 & 0 & 8 & 9 & 4.420 & 0.352 & -0.125 & 1 & 1 & 9 & 11 & 1.760 & 0.780 & -0.092 \\
\hline & Low & 0 & 2 & 13 & 15 & & & & 5 & 1 & 13 & 19 & & & \\
\hline & Total & 1 & 2 & 27 & 30 & & & & 7 & 2 & 24 & 33 & & & \\
\hline \multirow{4}{*}{ Rest } & Excellent & 0 & 0 & 10 & 10 & & & & 2 & 1 & 11 & 14 & & & \\
\hline & Moderate & 1 & 1 & 4 & 6 & 6.058 & 0.195 & -0.046 & 2 & 1 & 9 & 12 & 2.841 & 0.585 & -0.111 \\
\hline & Low & 0 & 1 & 13 & 14 & & & & 3 & 0 & 4 & 7 & & & \\
\hline & Total & 1 & 2 & 27 & 30 & & & & 7 & 2 & 24 & 33 & & & \\
\hline
\end{tabular}

$\chi^{2}$ : Chi-squared test, $\mathrm{p}$ : appearance probability, $\mathrm{r}^{\mathrm{k}}:$ Kendall rank correlation coefficient

*: $\mathrm{p}<0.05$

\section{Discussion}

Irregular life habits have been known to induce not only mental but also physical stress reactions (Takenaka.). Studies have shown that care workers work irregular hours (night shifts and overtime), have irregular life habits (meal times and bedtime), and have a wide range of work responsibilities (mobility, bathing, and toilet assistance) (Okamura et al., Ricardo et al.). Considering that care workers experience greater physical and mental burden, as well as stress, than general workers, their life habits can be easily disrupted. Nojima et al. reported that d-ROMs and BAP were associated with health conditions, life habits, and working conditions.

The present study examined the differences in physical characteristics, sleeping habits, health and life habits, and physiological stress (d-ROMs and BAP) among female care workers and general workers based on the assumption that care workers experience greater mental and physical stress than general workers. Although no differences in age and BMI had been observed between care workers and general workers, care workers were taller and heavier than general workers (Table 4). This may be explained by the need for a large physique and decent physical fitness when caring for individuals with physical difficulties (elderly and/or disabled) who require physical assistance, such as wheelchair-mobility, toilet and bathing assistance.

Given that care workers woke up later and slept longer than general workers (Table 4), the unique night and overtime work of care workers may affect their life habits (Ono et al.). Harano et al. reported that care workers recover from physical fatigue by taking naps during night shifts and taking adequate rest after the night shifts until the next working shift. The results of the present study showed that care workers had better physical fitness and greater sleep durations to recover from fatigue than general workers. Both care workers and general workers had average health and life habit parameters scores that fell within the "moderate" and "slightly low" categories, with no difference being observed between both groups (Table 5). The total health and life habits rating for both care workers and general workers indicated "caution required," which suggested an undesirable life habits, when health and life habit patterns were evaluated using the following four scales: (1) sufficient, (2) caution required-life habits, (3) caution required-health, and (4) caution required. 
Tokunaga, who evaluated the average health parameter score among 182 adult men and women aged 29 or younger and 108 adult men and women older than 30, found that their scores were "somewhat excellent," which indicated a "sufficient" total rating of health and life habits.

The present study found that both care workers and general workers in their 50s had poor health status and life habits. On the other hand, a considerable amount of time had elapsed since the study by Tokunaga, and many individuals in today's society may have more stressors.

The results for general workers might have been affected by the inclusion of a number of interpersonal relationships or assistance activities (see the Method section).

The present study showed that most of care workers and general workers had been rated as stressed $(90 \%$ and $73 \%$, respectively), while only a small number had normal d-ROMs levels. However, no significant difference in d-ROMs levels had been observed between both groups, thus average values among care workers and general workers suggested "high oxidative stress."

More than half of care workers and general workers (57\% and 61\%, respectively) had an average BAP value that fell within either optimum or borderline levels, with no significant difference between both groups. One study showed that those who live a well-regulated life usually have low d-ROMs and high BAP levels (Nagata.) and tend to be evaluated as being in physiologically good condition.

Given that d-ROMs levels of both care workers and general workers included herein were high, we can surmise that they had high oxidative stress and were under highly stressful conditions physiologically.

After examining 312 men and women (average age 36.7), Nojima et al. reported that d-ROMs were higher among women than men and that they increase with age. However, the same study showed that BAP exhibited no age- and sex-related differences.

Komatu et al. and Trevisan et al. have shown that d-ROMs levels are easily affected by hormones and that middle-aged and older women tend to have especially high d-ROMs levels due to irregular secretion of physiological hormones or menopause.

High d-ROMs levels promote a loss of balance in oxidant and antioxidant reactions in the body, resulting in life habit-related and many other diseases (Komatu et al., Kawakami et al.).

BAP, on the other hand, reflects the reducing ability of an organism, which prevents activities of reactive oxygen. Homeostasis is therefore maintained by the balance between d-ROMs and BAP.

As previously stated, living organisms have BAP, which counters d-ROMs and increases when the organism is exposed to high d-ROMs levels. However, an organism with physical disorders will eventually run out of BAP and does not function adequately even when d-ROMs levels are increased (Nojima et al.).

All subjects included in the present study were-middle-aged women around the age of 50 who were exposed to high d-ROMs. However, given that none of them had any physical disorders, it can be inferred their BAP functioned adequately.

In addition, because d-ROMs were measured during working hours, the increase of d-ROMs could have prevented the increase in BAP as a balancing mechanism to temporarily maintain homeostasis, thereby resulting in normal or decreased BAP levels.

Nonetheless, no significant difference in d-ROMs and BAP, which are indexes for physiological stress, had been found between both care workers and general workers, meaning that they were under similar stress levels.

We hypothesized that care workers would experience more health and life habit changes and physiological stress than general workers because of their irregular working hours and greater physical burden.

However, no significant differences in all health and life habit parameters or physiological stress indexes had been found between care workers and general workers, which do not support our hypothesis.

One possible reason could be that the general workers also had poor health, undesirable life habits, and high d-ROMs levels, similar to care workers.

Significant correlations were found between d-ROMs and weight $(r=0.439)$ and d-ROMs and BMI $(r=0.382)$ among care workers and between d-ROMs and weight $(r=0.377)$ and BAP and BMI $(r=-0.359)$ among general workers. This fact suggests that the possibility of relationship between obesity and stress.

BMI has been used as a simple index for obesity (Swinburn et al.), while d-ROMs has been generally positively correlated with obesity (Kozuka et al., Suzuki et al., Im et al.). Such findings are reflected in our results wherein 
d-ROMs was correlated with weight among care workers and general workers and with BMI among care workers, whereas BAP was correlated negatively with BMI among general workers (Table 6). In addition, among general workers, d-ROMs was significantly correlated with awakening time and bedtime, while no such correlation had been observed among care workers (Table 6).

We believe that the absence of a significant correlation between d-ROMs and awakening time and bedtime among care workers was due to their considerably greater life habits irregularity compared to general workers. General workers had shorter sleep durations than care workers (Table 6).

Although d-ROMs and BAP showed no significant relationship with health and life habit parameters among general workers, a significant relationship was found only between d-ROMs and movement among care workers. Among 27 subjects that fell within the "other (mild to very strong)" ratings of d-ROMs, $18(60 \%)$ had low movement parameter scores (Table 8).

The movement factor consists of two elements: exercise behavior/conditions (i.e., enforcement frequency of movement or sports) and awareness about exercise (i.e., the effect and enjoyment of movement or sports and the individual's will to start them). The present results suggest that many subjects with high d-ROMs do not get enough movement or do not expect to benefit from movement and believe that physiological stress is not related to health or life habits, such as diet and nutrition.

Future studies should compare physiological stress during working hours and non-working days between general workers not involved in interpersonal relationship or assistance activities and workers in similar fields as care workers, such as nurses.

\section{Conclusion}

Care workers were physically larger and slept longer, probably to recover from fatigue, than general workers. Both groups had high d-ROMs levels and normal or decreased BAP levels, with no significant difference in terms of either index. Hence, we inferred that both groups were chronically in stressful conditions. Notably, general workers were judged to have poor health and undesirable life habits, similar to care workers.

Moreover, several care workers with high d-ROMs levels tended to not participate in sports or exercise and did not to expect to profit from them. Because we measured physiological stress marker levels only during working hours to accommodate cooperation by both groups, the increase in d-ROMs levels could have prevented the increase in BAP as a balancing mechanism to temporarily maintain homeostasis, thereby resulting in normal or decreased BAP levels.

Measurements of the physical stress markers during holidays would distinguish the stress levels of care workers from those of general workers. In future studies, selecting workers with consistent working times as the general workers may help clarify differences between care workers and general workers. Despite the limitations of this study, we also found no correlation between d-ROMs levels and variations in awakening times and bedtimes of care workers. These results may be attributable to their irregular schedules comparison with those of general workers.

\section{References}

Alberti, A., bolognini, L., Macciantelli, D., et al. (2000). The radical cation of N, N-diethyl-para-phenyle ndiamine: a possible indicator of oxidative stress in biological samples. Res Chem Intermed, 26, 253-267. https://doi.org/10.1163/156856700X00769

Care Work Foundation. (2008). Survey of Welfare Service of Care Workers, Survey of Welfare Service of Care Workers working at care station in 2007.

Casado, A., Castellanos, A., López-Fernández, M. E., Ruiz, R., Imedio, E. L., ... Fernández-Nieto, A. M. (2011). Determination of oxidative and occupational stress in palliative care workers. Clinical Chemistry and Laboratory Medicine, 49(3), 471-477. https://doi.org/10.1515/CCLM.2011.061

Dohi, K., Satoh, K., Nakamachi, T., Yofu, S., Hiratsuka, K., Nakamura, S., ... Aruga, T. (2007). Does Edaravone (MCI-186) act as an antioxidant and a neuroprotector in experimental traumatic brain injury? Antioxid Redox Signal, 8, 281-287. https://doi.org/10.1089/ars.2007.9.281

Fukuda, S. (2015). Objective and subjective assessment for stress. Kansai University Welfare Sciences, Department of Welfare Sciences, 10, 23-28.

Harano, K., Taniguti, T., \& Kobayashi, H. (2012). Fatigue in night-duty professional caregivers working at a public long-term care facility for the elderly. Kawasaki Medical Welfare Journal, 21(2), 208-217. 
Hatakeyama, T., Nagano, M., \& Ohnuki, K. (2008). The relationship among the oxidative stress, biological antioxidant potency, lifestyle, and biochemical markers in healthy people. The Journal of Japan Mibyou System Association, 14(1), 6-11.

Hayashi, T., Kobayashi, S., Suzuki, Y., Sone, K., Nawai, K., Sawada, K., ... Fukaya, Y. (2011). Occupational stress of health professions on geriatric facilities: a comparison among rehabilitation staffs, nursing staffs, care-givers and social workers. Medical Health Studies Study the Second, 43-63.

Im, J. N., Jae, S. P., \& Sat, B. P. (2019). Association between Abdomial obesity and oxidative Stress in Korean Adults. Korean J Fam Med, 40(6), 395-398. https://doi.org/10.4082/kjfm.18.0086

Inaba, R., \& Inoue, M. (2011). Relationship between burnout and work-related stress among female nurses. Japanese Society of Occupational Medicine and Traumatology, 59(3), 129-136.

Kawakami, T., Yura, A., Ryu, K., Sambe, T., Ogawa, K., Inagaki, M., ... Iwai, S. (2014). The correlation between oxidative and lipids in blood. Showa Bachelor Journal, 7(4), 403-412.

Kawano, Y., Demura, S., Tanaka, Y., \& Matsuura, Y. (2017). Study on stress factors in care workers. International Journal of Nursing \& Care, 1(3), 1-8.

Komatu, F., Ksgawa, Y., Sakuma, M., et al. (2006). Investigation of oxidactive stress and dietary habits in Mongolian People, compared to Japanese people. Nutrition \& Metabolism, 3, 21. https://doi.org/10.1186/1743-7075-3-21

Kozuka, T., Okuhisa, H., \& Masuaki, H. (2012). Oxidative stress and metabolic syndrome. Hormone Frontier in Gynecology, 19(2), 125-130.

Kuragaki, H. (2015). Wismerll News Interview Report, 59, 124-126.

Kwiatkowski. (2011). Effects on careers caring for residents who are limited in their everyday capabilities. A comparison of several measuring points in a pilot study. Pflege Z, 64(5), 286-290.

Maslach, C. (1993). Burnout: A multidimensional perspective. In W. B. Schaufeli, C. Maslach, \& T. Marek (Eds.), Professional burnout: Recent developments in theory and research. Washington, DC: Taylor \& Francis.

Mimura, K., Kobayashi, T., \& Mizukoshi, S. (2007). A Study of Quantification of Oxidative Stresses Caused by Lifestyle Habits. The Official Journal of Japanese Society of Laboratory Medicine, 55(1), 35-40.

Ministry of Health. (2005). Survey on Employment Trends in 2004 for regular workers. Care Work Foundation: Survey of Care Workers Welfare Service in 2004.

Ministry of Health. (2017). Occupational health and safety survey, Fact-F finding in 2016.

Ministry of Health. (2018). National Health and Nutrition Survey in 2017.

Nagata, K., Hasegawa, T., Hirokado, Y., Kiyama, K., \& Otsuki, C. (2008). Lifestyle-related Diseases and the Oxidative Stress Regulation System. Japnese Society of Psychosomatic Medicine, 48, 177-183.

Nakao, H., Kobayashi, T., \& Shinagawa, S. (2003). Relationships between work-related stress, depression symptoms and lifestyle of nurses. School of Nursing, Yamaguchi Prefectural University Bulletin, 7.

Nara, S., \& Suwa, K. (2007). A consideration for continuance of care works by nursing worker's. Meisei University Bulletin of Education and Research, 22, 34-43.

Nojima, J., Miyagaea, M., Kodama, M., Kimoto, Y., Tsuneoka, H., Ichihara, K., \& Hinoda, H. (2010). Measurement of oxidative stress level by automatic analyzer BM-1650. Medical examination, 59(3), 199-207.

Okamura, H., Tsuda, A., Yajima, J., Mark, H., Horiuchi, S., Toyoshima, N., \& Matsuishi, T. (2010). Short sleeping time and psychobiological responses to acute stress. International Journal of Psychophysiol, 78(3), 209-214. https://doi.org/10.1016/j.ijpsycho.2010.07.010

Okazaki, H., Nakamura, M., \& Kinoshita, M. (2012). Effectiveness of learning sessions for improving lifestyle of nursing and care staff.

Ono, S., Komada, Y., Ariga, M., Tsutsumi, H., \& Shirakawa, S. (2005). An epidemiological study of the relationship between bowel movement habits and sleep health of adult women living in Tokyo metropolitan area. Journal of Japanese Society of Psychosomatic Obstetrics and Gynecology, 10(2), 67-75.

Onoda, Y., \& Shimomitu, T. (2006). Preventing mental health deficiencies in the workplace, evaluation of 
workplace stress and utilization of that in the field. Japanese Journal of Psychosomatic Internal Medicine, $10(1), 18-22$.

Ricardo, J. S., Cartner, L., Oliver, S. J., Laing, S. J., Walters, R., Bilzon, J. L., \& Walsh, N. P. (2009). No of a 30-h period of sleep deprivation on leukocyte trafficking, neutrophil degranulation and saliva IgA responses to exercise. Eur. J. Appl. Physiol, 105(3), 499-504. https://doi.org/10.1007/s00421-008-0931-3

Seki, Y. (2009). Evaluation of total oxidative stress by d-ROMs testing. Biological Sample Analysis, 32(4), 301-306.

Shimomitu, T. (2004). Questionnaire of simple occupational stress. Japanese Journal of Occupational Mental Health, 1(2), 25-36.

Suzuki, K., Ito, Y., Chiai, J., Kusuhara, Y., Hashimoto, S., Tokudome, S., et al. (2003). Relationship between obesity and serum markers of oxidative stress and inflammation in Japanese. Asian Pacific Journal of Cancer Prevention, 4(3), 259-266.

Swinburn, B. A., Craing, P. L., Daniel, R., et al. (1996). Body composition differences between Polynesians and Caucasians assessed by bioelectrical impedance. International Journal of Obese, 20, 889-894.

Takenaka, S. (2005). Stress management. Yumani Shobo, 3.

Tokunaga, M. (2003). Diagnostic Inventory of Health and Life Habit 2, (Junior high school students-adults). Yoyo Physical, 1-32.

Trevisan, M., Browne, R., \& Ram, M., et al. (2001). Corrlates of markers of oxidative status in the general population. American Journal of Epidemiology, 154(4), 348-356. https://doi.org/10.1093/aje/154.4.348

Wayner, D. D., Burton, G. W., Ingoid, K. U., et al. (1985). Quantitative measurement of the total, peroxyl radical-trapping antioxidant capability of human blood plasma by controlled peroxidation. The important contribution made by plasma Proteins. FEBS Lett, 187, 33-37. https://doi.org/10.1016/0014-5793(85)81208-4

\section{Copyrights}

Copyright for this article is retained by the author(s), with first publication rights granted to the journal.

This is an open-access article distributed under the terms and conditions of the Creative Commons Attribution license (http://creativecommons.org/licenses/by/4.0/). 